\title{
General expressions for the stress intensity factor of a one-point bend beam
}

\author{
I. Villa *, J.A. Loya, J. Fernández-Sáez \\ Department of Continuum Mechanics and Structural Analysis, University Carlos III of Madrid, \\ Avda. de la Universidad 30, 28911 Leganés, Madrid, Spain
}

\begin{abstract}
The aim of this work is to find general expressions to determine the stress intensity factor of a one point bend beam like specimen, whether from the measurement of the applied load or the crack mouth opening displacement. The expressions, obtained by applying the superposition principle, involve the decomposition of the general case into three auxiliary problems. The solution of two of them (pure bending and three point bending) is well known, while the solution of the third (one point bending) is developed in the present work. The proposed expressions are compared to numerical results obtained by the finite element method and their accuracy is equal to or better than available expressions published elsewhere.
\end{abstract}

Keywords: Stress intensity factor; CMOD; One point bending

\section{Introduction}

Impact testing of beam-like pre-cracked specimens is the usual method to determine the dynamic fracture toughness of materials. The well-known Charpy impact test is probably the most popular procedure [1], but other arrangements have been devised for high strain rate conditions [2 5$]$.

Experimental [6] and numerical analyses [7] have shown that in this kind of tests the specimen may lose contact with the supports during the initial stage of loading and is then entirely loaded by inertial forces. This loading condition is known as one-point bending (OPB) and is the initial stage of any conventional three-point bending (TPB) impact test.

Several authors have proposed OPB impact experiments (tests on unsupported specimens) applied to different pre-cracked specimen geometries such as beams [8 11], C-shaped specimens [12], compact compression specimens [11,13] and notched plates [14].

\footnotetext{
* Corresponding author. Tel.: +34 916249162; fax: +34 916249430.

E mail address: ivilla@ing.uc3m.es (I. Villa).
} 


\section{Nomenclature}

a crack length

$B$ thickness of the specimen

$E \quad$ Young's modulus of the material

$E^{\prime} \quad$ generalized Young's modulus of the material

$F(t) \quad$ general force acting on an elastic specimen

$h_{k}(\mathrm{t}) \quad$ stress intensity factor corresponding to a unit impulse

$h_{\Phi} \quad$ response function corresponding to a unit impulse

$k_{\mathrm{d}} \quad$ constant which is assumed to be the same as in a static TPB test

$K_{\text {Id }} \quad$ dynamic fracture toughness

$K_{\mathrm{I}}^{\mathrm{s}} \quad$ stress intensity factor due to a unit uniformly-distributed volume force

$K_{\mathrm{I}}(t) \quad$ stress intensity factor

$M \quad$ bending moment in the central cross-section of the original beam

$M_{1} \quad$ bending moment in the central cross-section due to a pure bending state

$M_{2}$ bending moment in the central cross-section due to a TPB state

$M_{3} \quad$ bending moment in the central cross-section due to an OPB state

$p_{\beta}(\alpha) \quad$ general cubic polynomial

$p_{4}(\alpha) \quad$ cubic polynomial corresponding to a TPB state of a specimen with $\beta \quad 4$

$p_{\infty}(\alpha) \quad$ cubic polynomial corresponding to a pure bending state

$p_{4}^{*}(\alpha) \quad$ cubic polynomial corresponding to an OPB state of a specimen with $\beta \quad 4$

$P \quad$ net external applied load

$q \quad$ uniformly distributed load

$q_{\beta}(\alpha) \quad$ general polynomial

$q_{4}(\alpha)$ polynomial corresponding to a TPB state

$q_{\infty}(\alpha)$ polynomial corresponding to a pure bending state

$q_{4}^{*}(\alpha)$ polynomial corresponding to an OPB state

$Q_{1} \quad$ external shear forces

$S \quad$ span of the specimen

$t_{\mathrm{f}} \quad$ instant at which the crack begins to grow

$u_{\mathrm{F}}(1 / 2, t)$ displacement of the central cross-section of the specimen

$u_{\mathrm{F}}(0, t)$ displacement of the end of the specimen

$W \quad$ width of the specimen

$\alpha \quad$ crack-to-width ratio

$\beta \quad$ span-to-width ratio

$\delta \quad$ crack mouth opening displacement

$\Phi(t) \quad$ generic variable

$\eta_{i} \quad$ weight coefficient proportional to the $i$ th symmetric mode contribution

$\lambda_{\beta}^{*}(\alpha) \quad$ relationship between $\psi^{*} \beta(\alpha)$ and $v^{*} \beta(\alpha)$

$\Lambda_{\beta}^{*}(\alpha)$ percentage difference between the value of $\lambda_{\beta}^{*}(\alpha)$ of an OPB specimen obtained by the FEM and different analytical expressions

$\mu \quad$ Poisson's ratio of the material

$\sigma_{n} \quad$ reference stress

$v_{\beta}^{*}(\alpha) \quad$ general dimensionless function corresponding to an OPB state

$v_{\infty}(\alpha)$ dimensionless function corresponding to a pure bending state

$v_{4}(\alpha) \quad$ dimensionless function corresponding to a TPB state of a specimen with $\beta \quad 4$

$v_{4}^{*}(\alpha) \quad$ dimensionless function corresponding to an OPB state of a specimen with $\beta \quad 4$

$v_{\beta}(\alpha) \quad$ general dimensionless function

$\omega_{i} \quad i$ th non-trivial eigenfrequency of the unsupported specimen

$\psi_{\beta}^{*}(\alpha)$ general dimensionless function corresponding to an OPB state 
$\psi_{\infty}(\alpha)$ dimensionless function corresponding to a pure bending state

$\psi_{4}(\alpha) \quad$ dimensionless function corresponding to a TPB state of a specimen with $\beta \quad 4$

$\psi_{4}^{*}(\alpha)$ dimensionless function corresponding to an OPB state of a specimen with $\beta \quad 4$

$\psi_{\beta}(\alpha)$ general dimensionless function

$\Psi_{\beta}^{*}(\alpha)$ percentage difference between the value of $\psi_{\beta}^{*}(\alpha)$ of an OPB specimen obtained by the FEM and analytical expressions

However, to obtain the dynamic fracture toughness, $K_{\mathrm{Id}}$, it is necessary to determine both the temporal evolution of the stress intensity factor (SIF) during the specimen loading process and the instant, $t_{\mathrm{f}}$, at which the crack begins to grow. Thus, $K_{\text {Id }}$ may be obtained as the SIF at the crack growth initiation time:

$$
K_{\mathrm{Id}}=K_{\mathrm{I}}\left(t_{\mathrm{f}}\right)
$$

Although full numerical procedures based on the finite element method (FEM) and complex experimental techniques are available to evaluate the temporal evolution of the SIF, simplified procedures have been proposed [15 19].

For an elastic specimen loaded by a concentrated force, $F(t)$, the general solution for any generic variable $\Phi(t)$ can be obtained by the convolution integral:

$$
\Phi(t)=\int_{0}^{t} F(\tau) h_{\Phi}(t-\tau) \mathrm{d} \tau
$$

$h_{\Phi}$ being the response function corresponding to a unit impulse applied at the same point and in the same direction as $F(t)$.

Kishimoto et al. [15] applied the above formulation to calculate the displacements in an OPB beam-like precracked specimen. The corresponding response function was obtained by modal analysis, considering the specimen as an Euler-Bernoulli cracked beam. They calculated the temporal evolution of the SIF as:

$$
K_{\mathrm{I}}(t)=k_{\mathrm{d}}\left(u_{\mathrm{F}}\left(\frac{1}{2}, t\right)-u_{\mathrm{F}}(0, t)\right)
$$

where the term in brackets indicates the difference between the displacement of the central cross-section of the specimen, $u_{\mathrm{F}}\left(\frac{1}{2}, t\right)$, and the displacement of the end of the specimen, $u_{\mathrm{F}}(0, t)$, and $k_{\mathrm{d}}$ is a constant which is assumed to be the same as in a static TPB test.

Rokach [16] used Eq. (2) to obtain the SIF directly:

$$
K_{\mathrm{I}}(t)=\int_{0}^{t} F(\tau) h_{k}(t-\tau) \mathrm{d} \tau
$$

For the response function $h_{k}$ (SIF corresponding to a unit impulse applied to an unsupported specimen), Rokach [16] derived the following expression:

$$
h_{k}(t)=K_{\mathrm{I}}^{\mathrm{s}} \sum_{i}^{\infty} \eta_{i} \omega_{i} \sin \omega_{i} t
$$

in which $K_{\mathrm{I}}^{\mathrm{s}}$ is the SIF due to a unit uniformly-distributed volume force, $\eta_{i}$ is the weight coefficient proportional to the $i$ th symmetric mode contribution, and $\omega_{i}$ is the $i$ th non-trivial eigenfrequency of the unsupported specimen.

Rokach [17] gives empirical formulae for the parameters $K_{\mathrm{I}}^{\mathrm{s}}, \eta_{i}$ and $\omega_{i}$ by fitting the results of 2D numerical analysis of different values of crack length and span-to-width ratio.

Other investigators [18,19], obtained the SIF in dynamic bending fracture tests from direct measurement of the crack mouth opening displacement (CMOD), applying the relationship between these variables corresponding to TPB. However, it was later shown [5] that in many cases the TPB conditions no longer hold, and OPB conditions must be considered. 
To the authors' knowledge, no general expression has been developed for the relationship between the SIF and CMOD, strictly applicable to OPB conditions.

The aim of this work is to find general expressions to determine the SIF of an OPB beam-like specimen, whether from the measurement of the applied load or the CMOD.

Based on the superposition principle, the solution method involves the decomposition of the general case into three auxiliary problems. The solution of two of them is well known, while that of the third is developed here.

A similar procedure was applied by Guinea et al. [20] to determine the SIF, compliance, and CMOD for a general TPB beam.

\section{Problem formulation}

Consider a general OPB beam-like specimen subjected to a uniformly distributed load of magnitude $q$, such as that depicted in Fig. 1(a), where $S$ is the span of the specimen, $W$ is the width, $B$ the thickness and $a$ is the crack length. Let $\alpha$ and $\beta$ be the crack-to-width ratio and span-to-width ratio:

$$
\alpha=\frac{a}{W} ; \quad \beta=\frac{S}{W}
$$

It is possible to decompose this general case by superposition, as is shown in Fig. 1(b), where a beam of span $\hat{\mathrm{S}}$ is isolated by introducing external shear forces, $Q_{1}$, and bending moments, $M_{1}$.

With this decomposition, the bending moment in the central cross-section of the original beam, $M$, may be rewritten as the sum of the bending moment in the central cross-section of a beam of span $\hat{\mathrm{S}}$ due to a pure bending state, $M_{1}$; a TPB state, $M_{2}$; and an OPB state, $M_{3}$, i.e.:

$$
M=\frac{q S^{2}}{8}=\frac{P S}{8}=M_{1}+M_{2}+M_{3}
$$

in which $P \quad q S$ is the net external applied load.

To maintain static equilibrium in the specimen, the external shear forces, $Q_{1}$, and the bending moment in the central cross-section due to the three states indicated in Fig. 1(b) are:

$$
\begin{aligned}
& Q_{1}=\frac{q(S-\hat{S})}{2} \\
& M_{1}=\frac{q}{8}(S-\hat{S})^{2}=\frac{q S^{2}}{8}\left(1-\frac{\hat{\beta}}{\beta}\right)^{2}=M\left(1-\frac{\hat{\beta}}{\beta}\right)^{2}
\end{aligned}
$$

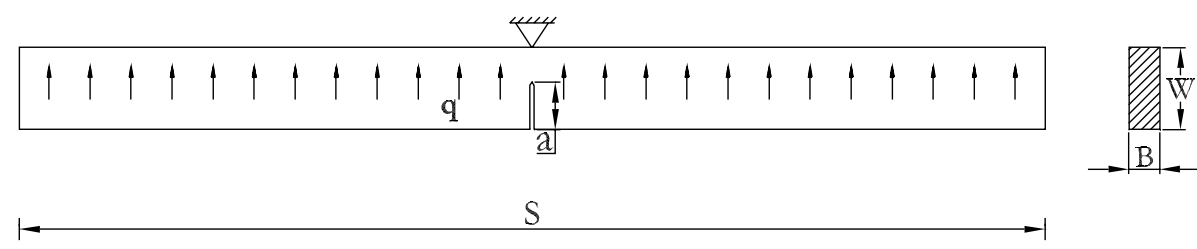

Fig. 1(a). OPB beam like specimen with an arbitrary $S / W$ ratio.

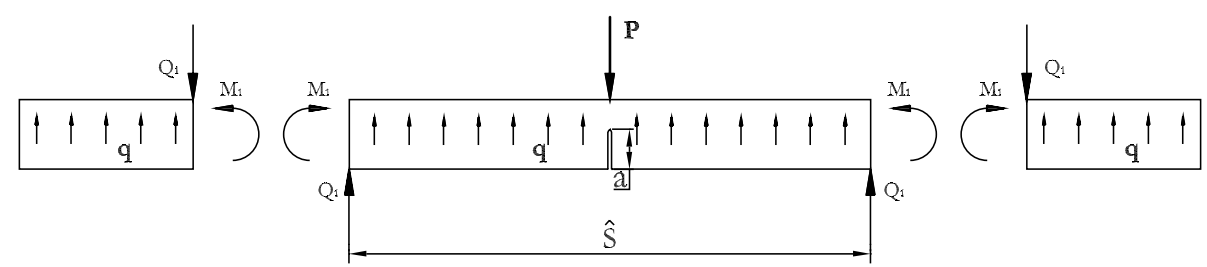

Fig. 1(b). Decomposition of the general case into three auxiliary problems. 


$$
\begin{aligned}
& M_{2}=\frac{q(S-\hat{S})}{2} \frac{\hat{S}}{2}=2 M\left(1-\frac{\hat{\beta}}{\beta}\right) \frac{\hat{\beta}}{\beta} \\
& M_{3}=\frac{q \hat{S}^{2}}{8}=\frac{q S^{2}}{8}\left(\frac{\hat{S}}{S}\right)^{2}=M\left(\frac{\hat{\beta}}{\beta}\right)^{2}
\end{aligned}
$$

in which $\hat{\beta}=\hat{S} / W$ is the span-to-width ratio related to a beam of span $\hat{S}$.

In the following sections, the above-mentioned decomposition is used to obtain general expressions for the SIF of an OPB beam-like specimen from the measurement of the external applied load, or from the measurement of the CMOD.

\section{General expression for the SIF from the applied load}

Starting from the expression originally proposed by Tada et al. [21] for TPB specimens, the SIF corresponding to an OPB beam-like specimen (Fig. 1(a)), $K_{\mathrm{I}}^{\mathrm{s}}$, may be expressed as a function of its geometry and a reference stress, $\sigma_{n}$, in the following way:

$$
K_{\mathrm{I}}^{\mathrm{s}}=\sigma_{n} \sqrt{ } W \psi_{\beta}^{*}(\alpha)=\frac{6 M}{B W^{3 / 2}} \psi_{\beta}^{*}(\alpha)
$$

where $\psi_{\beta}^{*}(\alpha)$ is a dimensionless function to be determined, and $M$ is the bending moment in the central crosssection, given by Eq. (7).

By applying the superposition principle, the SIF of the original specimen may be written as the sum of the contributions of the three states indicated in Fig. 1(b). Considering $\hat{\beta}=4$, Eq. (12) will be:

$$
K_{\mathrm{I}}^{\mathrm{s}}=\frac{6}{B W^{3 / 2}}\left[M_{1} \psi_{\infty}(\alpha)+M_{2} \psi_{4}(\alpha)+M_{3} \psi_{4}^{*}(\alpha)\right]
$$

where $\psi_{\infty}(\alpha)$ is the dimensionless function of a pure bending state, $\psi_{4}(\alpha)$ is the dimensionless function of a TPB state of a specimen with a span-to-width ratio of 4 , and $\psi_{4}^{*}(\alpha)$ is the dimensionless function of an OPB state of a specimen with a span-to-width ratio equal to 4 .

Placing now Eqs. (9) (11) into Eq. (13) and making Eq. (12) equal to Eq. (13), the following expression is obtained for $\psi_{\beta}^{*}(\alpha)$ after some algebra:

$$
\psi_{\beta}^{*}(\alpha)=\psi_{\infty}(\alpha)+\frac{4}{\beta}\left[2 \psi_{4}(\alpha)-2 \psi_{\infty}(\alpha)\right]+\left(\frac{4}{\beta}\right)^{2}\left[\psi_{\infty}(\alpha)-2 \psi_{4}(\alpha)+\psi_{4}^{*}(\alpha)\right]
$$

Approximate expressions for $\psi_{\beta}$ has been given in the following general form [20]:

$$
\psi_{\beta}(\alpha)=\frac{\sqrt{ } \alpha}{(1-\alpha)^{3 / 2}(1+3 \alpha)} p_{\beta}(\alpha)
$$

in which $p_{\beta}(\alpha)$ is a cubic polynomial. For $\beta \quad 4$ and $\beta \rightarrow \infty$ (pure bending), they are [20]:

$$
\begin{aligned}
& p_{4}(\alpha)=1.90+0.41 \alpha+0.51 \alpha^{2}-0.17 \alpha^{3} \\
& p_{\infty}(\alpha)=1.99+0.83 \alpha-0.31 \alpha^{2}+0.14 \alpha^{3}
\end{aligned}
$$

However, to apply Eq. (12) to determine $K_{\mathrm{I}}^{\mathrm{s}}$ for a general OPB beam-like specimen, $\psi_{4}^{*}(\alpha)$ must be developed (Eq. (14)). We use an expression analogous to Eq. (15) to find the polynomial $p_{4}^{*}(\alpha)$, corresponding to an OPB test of a beam with a span-to-width ratio equal to 4 , i.e.:

$$
\psi_{4}^{*}(\alpha)=\frac{\sqrt{ } \alpha}{(1-\alpha)^{3 / 2}(1+3 \alpha)} p_{4}^{*}(\alpha)
$$

With this arrangement, it is possible to rewrite Eq. (14) as follows:

$$
\psi_{\beta}^{*}(\alpha)=\frac{\sqrt{ } \alpha}{(1-\alpha)^{3 / 2}(1+3 \alpha)}\left\{p_{\infty}(\alpha)+\frac{4}{\beta}\left[2 p_{4}(\alpha)-2 p_{\infty}(\alpha)\right]+\left(\frac{4}{\beta}\right)^{2}\left[p_{\infty}(\alpha)-2 p_{4}(\alpha)+p_{4}^{*}(\alpha)\right]\right\}
$$




\subsection{Determination of $p_{4}^{*}(\alpha)$}

In the case of an OPB beam-like specimen with a span-to-width ratio of $4, K_{\mathrm{I}}^{\mathrm{s}}$ may be rewritten, in accordance with Eq. (12) and Eq. (18), as follows:

$$
K_{\mathrm{I}}^{\mathrm{s}}=\frac{6 M}{B W^{3 / 2}} \frac{\sqrt{ } \alpha}{(1-\alpha)^{3 / 2}(1+3 \alpha)} p_{4}^{*}(\alpha)
$$

To find $p_{4}^{*}(\alpha)$, numerical simulations were made to determine the SIF of an OPB beam-like specimen with a span-to-width ratio equal to 4 , using the finite element method (FEM) implemented in the commercial code ABAQUS/Standard [22], for 17 different crack-to-width ratios ranging from 0.10 to 0.90 in steps of 0.05 .

Given the symmetry of the problem, only half the specimen was analyzed using four-node quadrilateral elements (Fig. 2(a)). Special attention was paid to the discretization near the crack tip, to take into account the stress and strain gradients present in this region.

In order to minimize the influence of the mesh density, a refined mesh zone composed of 800 elements was moved, without variations of its shape or size, in all the cases analyzed, being always in the same position relative to the crack tip. Fig. 2(b) shows in detail the meshes used in the discretization near the crack tip. Depending on the crack-to-width ratio considered, the number of elements varied between 2640 and 3600, and the number of nodes between 2734 and 3713.

Additional computations were performed with eight-node quadrilateral elements and various mesh refinements to verify convergence and accuracy. Moreover, the results obtained for TPB with these meshes agree with those published by Guinea et al. [20].

The SIF was determined from the values of $J$ integral provided by the finite element code ABAQUS/Standard [22], using a procedure based on the virtual crack extension/domain integral methods for such calculations $[23,24]$. This method is simple to use and provides excellent accuracy, even with rather coarse meshes, so singular elements are not required.

In a linear elastic case, the relation between the $J$ integral and the SIF is given by:

$$
K_{\mathrm{I}}^{\mathrm{s}}=\sqrt{ } E^{\prime} J
$$

where $E^{\prime}$ is the generalized Young's modulus of the material (equal to $E$ for plane stress and $E /\left(1-v^{2}\right)$ for plane strain, in which $v$ is the Poisson's ratio of the material).

The numerical analysis were performed in plane strain conditions, but the results corresponding to the SIF agree with those obtained using plane stress elements.

From these results, 17 individual values of $p_{4}^{*}$ were determined by means of Eq. (20) and then, by the least square method, the following expression was obtained for $p_{4}^{*}(\alpha)$ :

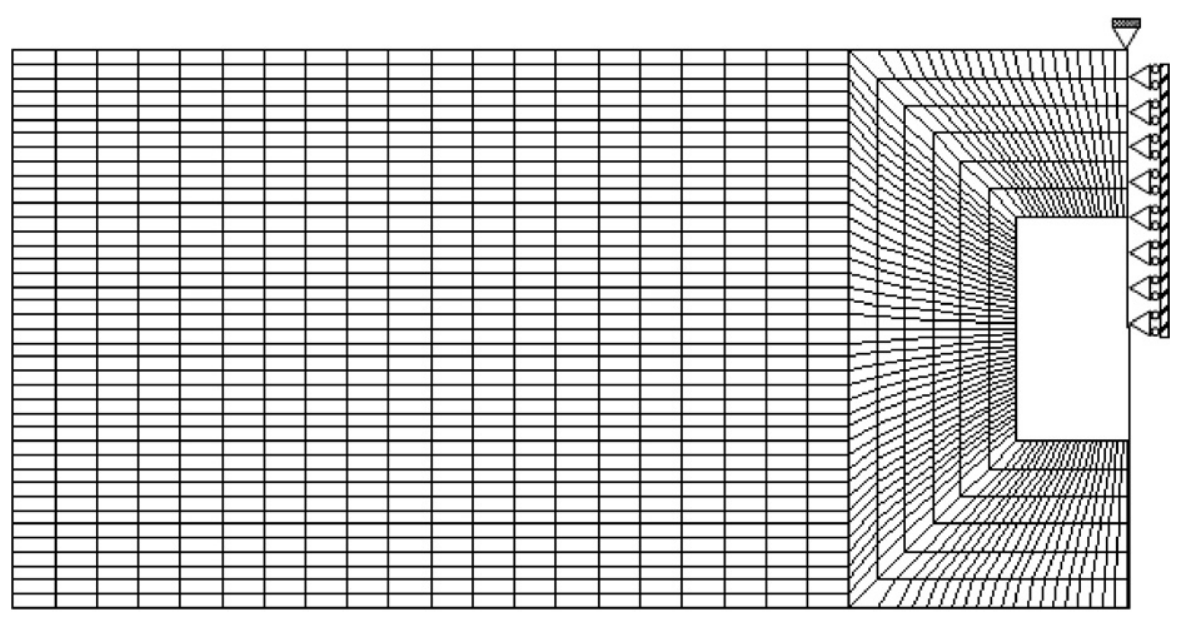

Fig. 2(a). Example of FEM meshes used. 


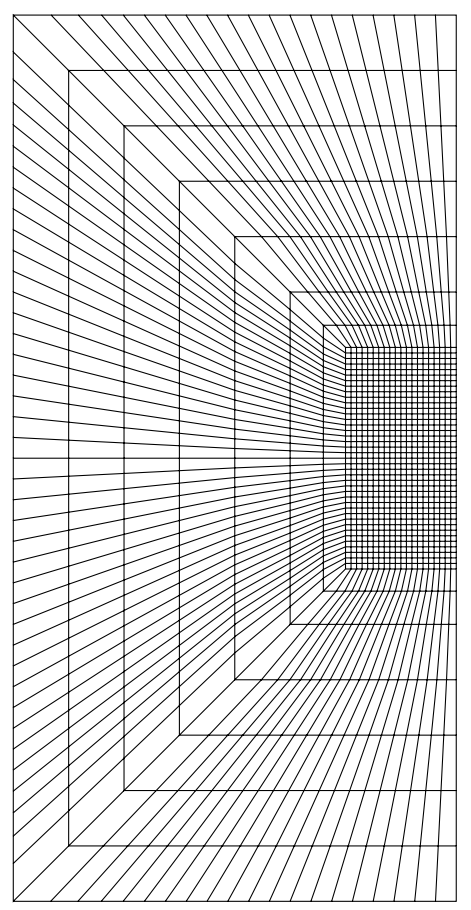

Fig. 2(b). Detail of the specimen mesh refined near the crack tip.

$$
p_{4}^{*}(\alpha)=1.72+0.23 \alpha+1.04 \alpha^{2}-0.39 \alpha^{3}
$$

Fig. 3 shows the accuracy of the proposed expression, with the differences from the numerical results obtained by applying the finite element method below $0.3 \%$ for $\alpha$ ranging between 0.10 and 0.90 .

Once the polynomial $p_{4}^{*}(\alpha)$ is determined, $\psi_{4}^{*}(\alpha)$ may be calculated by Eq. (18), and then the SIF from the external applied load of a general beam-like specimen may be obtained by applying successively Eqs. (14) and (12).

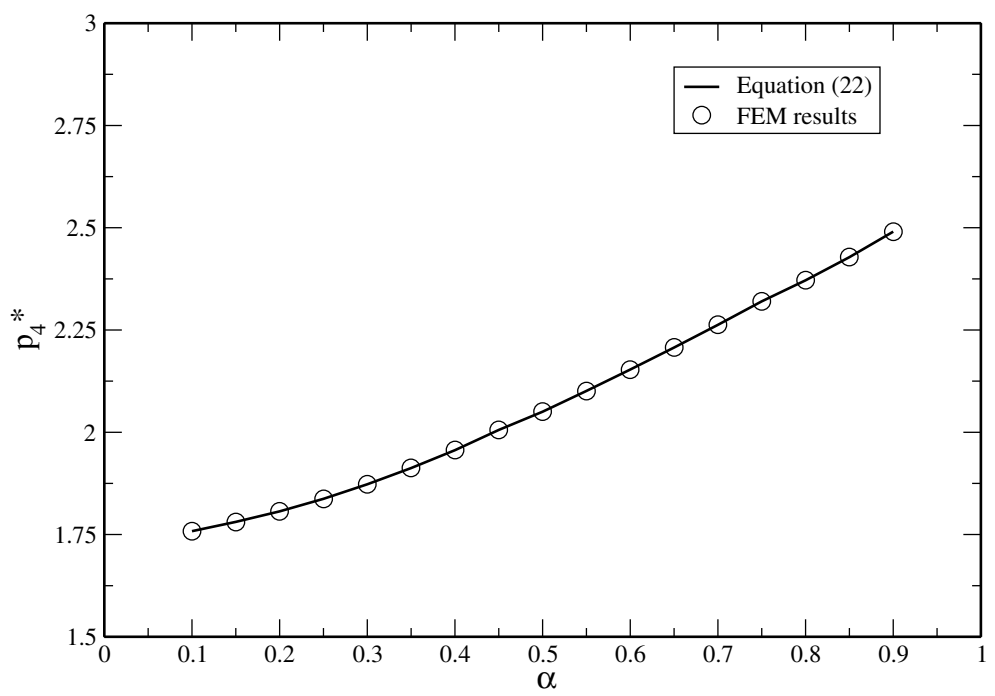

Fig. 3. Polynomial fitting for parameter $p_{4}^{*}(\alpha)$. 


\section{General expression of the SIF from the CMOD}

As in the previous case, using the expression proposed by Tada et al. [21] for TPB conditions, in a cracked OPB specimen such as that depicted in Fig. 1(a), the CMOD, $\delta$, may be written as follows:

$$
\delta=\frac{4 \sigma_{n} a}{E^{\prime}} v_{\beta}^{*}(\alpha)=\frac{24 M a}{B W^{2} E^{\prime}} v_{\beta}^{*}(\alpha)
$$

$v_{\beta}^{*}(\alpha)$ being a dimensionless function to be determined.

Working out $M$ in Eq. (23) and setting this value into Eq. (12), the following relationship between SIF and CMOD is obtained:

$$
K_{\mathrm{I}}^{\mathrm{s}}=\frac{\delta E^{\prime} \sqrt{ } W}{4 a} \lambda_{\beta}^{*}(\alpha)
$$

where

$$
\lambda_{\beta}^{*}(\alpha)=\frac{\psi_{\beta}^{*}(\alpha)}{v_{\beta}^{*}(\alpha)}
$$

The previous expression, very useful to determine the SIF from experimental values of the CMOD, is defined as a function of $\psi_{\beta}^{*}(\alpha)$ and another general expression, $v_{\beta}^{*}(\alpha)$, that needs to be determined.

Applying the procedure indicated in Section 3, the following expression, formally identical to Eq. (14), is obtained for $v_{\beta}^{*}(\alpha)$ :

$$
v_{\beta}^{*}(\alpha)=v_{\infty}(\alpha)+\frac{4}{\beta}\left[2 v_{4}(\alpha)-2 v_{\infty}(\alpha)\right]+\left(\frac{4}{\beta}\right)^{2}\left[v_{\infty}(\alpha)-2 v_{4}(\alpha)+v_{4}^{*}(\alpha)\right]
$$

where $v_{\infty}(\alpha)$ is the dimensionless function of a pure bending state, $v_{4}(\alpha)$ is the dimensionless function of a TPB state of a specimen with a span-to-width ratio of 4 , and $v_{4}^{*}(\alpha)$ is the dimensionless function corresponding to an OPB state of a specimen with a span-to-width ratio equal to 4 .

Approximate expressions were found in the literature [21] for $v_{\beta}(\alpha)$ with the following form:

$$
v_{\beta}(\alpha)=q_{\beta}(\alpha)+\frac{0.66}{1-\alpha^{2}}
$$

in which $q_{\beta}(\alpha)$ is a polynomial. For $\beta \quad 4$ and $\beta \rightarrow \infty$ (pure bending), they are [21]:

$$
\begin{aligned}
& q_{4}(\alpha)=0.76-2.28 \alpha+3.87 \alpha^{2}-2.04 \alpha^{3} \\
& q_{\infty}(\alpha)=0.80-1.70 \alpha+2.40 \alpha^{2}
\end{aligned}
$$

In order to determine $K_{\mathrm{I}}^{\mathrm{s}}$ for a general OPB beam-like specimen by applying Eq. (24), it is necessary to develop the polynomial $v_{4}^{*}(\alpha)$, corresponding to an OPB state of a specimen with a span-to-width ratio equal to 4 , using an expression analogous to Eq. (27) to find the polynomial $q_{4}^{*}(\alpha)$, i.e.:

$$
v_{4}^{*}(\alpha)=q_{4}^{*}(\alpha)+\frac{0.66}{1-\alpha^{2}}
$$

With this arrangement, it is possible to rewrite Eq. (26) as follows:

$$
v_{\beta}^{*}(\alpha)=q_{\infty}(\alpha)+\frac{4}{\beta}\left[2 q_{4}(\alpha)-2 q_{\infty}(\alpha)\right]+\left(\frac{4}{\beta}\right)^{2}\left[q_{\infty}(\alpha)-2 q_{4}(\alpha)+q_{4}^{*}(\alpha)\right]+\frac{0.66}{1-\alpha^{2}}
$$

\subsection{Determination of $q_{4}^{*}(\alpha)$}

In an OPB test of a specimen with a span-to-width ratio equal to 4, the CMOD may be expressed, in accordance with Eq. (23), as follows: 


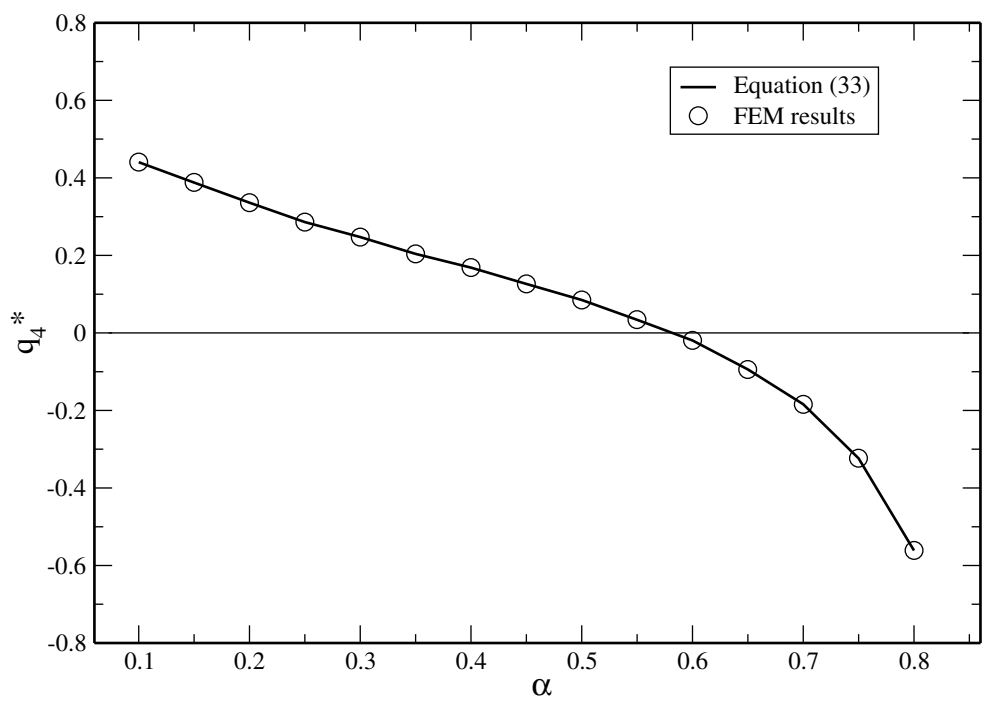

Fig. 4. Polynomial fitting for parameter $q_{4}^{*}(\alpha)$.

$$
\delta=\frac{24 M a}{B W^{2} E^{\prime}}\left[q_{4}^{*}(\alpha)+\frac{0.66}{1-\alpha^{2}}\right]
$$

$q_{4}^{*}(\alpha)$ being a polynomial to be determined.

We used the results of the finite element computations to determine $p_{4}^{*}(\alpha)$ but only in the range $0.10 \leqslant \alpha \leqslant 0.80$ because if the crack length is longer $(\alpha>0.80)$, the behaviour of the CMOD changes and it is very difficult to cover the whole $\alpha$ range with the same expression and reasonably good agreement.

The CMOD was obtained from the displacement of the node at the end of the edge crack and then, from Eq. (32), the values of $q_{4}^{*}$ were obtained.

Fitting these results by the least square method, the following expression was obtained for $q_{4}^{*}(\alpha)$ :

$$
q_{4}^{*}(\alpha)=0.66-3.21 \alpha+13.65 \alpha^{2}-38.30 \alpha^{3}+52.67 \alpha^{4}-28.53 \alpha^{5}
$$

Fig. 4 shows the agreement between the numerical results and the expression proposed, with the same accuracy as in the case of $p_{4}^{*}(\alpha)$.

Once the polynomial $q_{4}^{*}(\alpha)$ is determined, $v_{4}^{*}(\alpha)$ may be calculated by means of Eq. (30). After that, the SIF from the CMOD for a general beam-like specimen can be obtained by applying successively Eqs. (26), (25) and, finally, Eq. (24).

\section{Validation of the general expressions}

We compared the values obtained by the proposed expressions of the SIF to the corresponding numerical results of specimens with different span-to-width ratio $\left(\begin{array}{lllll}\beta & 2.5, \beta & 4, \beta & 5.5 \text { and } \beta & 8\end{array}\right)$ and crack-to-width ratio $(\alpha \quad 0.10$ to $\alpha$ 0.90), computed by the FEM.

\subsection{SIF from the measurement of the applied load}

The function $\psi_{\beta}^{*}(\alpha)$ with which the SIF is calculated from the applied load (see Section 3), was compared to the values of $\psi_{\beta}^{*}(\alpha)$ obtained from the results presented by Rokach [17] for OPB specimens, and from those calculated by Guinea et al. [20] for TPB specimens.

Fig. 5 shows, for $\beta \quad 2.5, \beta \quad 4, \beta \quad 5.5$ and $\beta \quad 8$, the percentage difference, $\Psi_{\beta}^{*}(\alpha)$, between the value of $\psi_{\beta}^{*}(\alpha)$ of an OPB specimen obtained by the FEM and: 

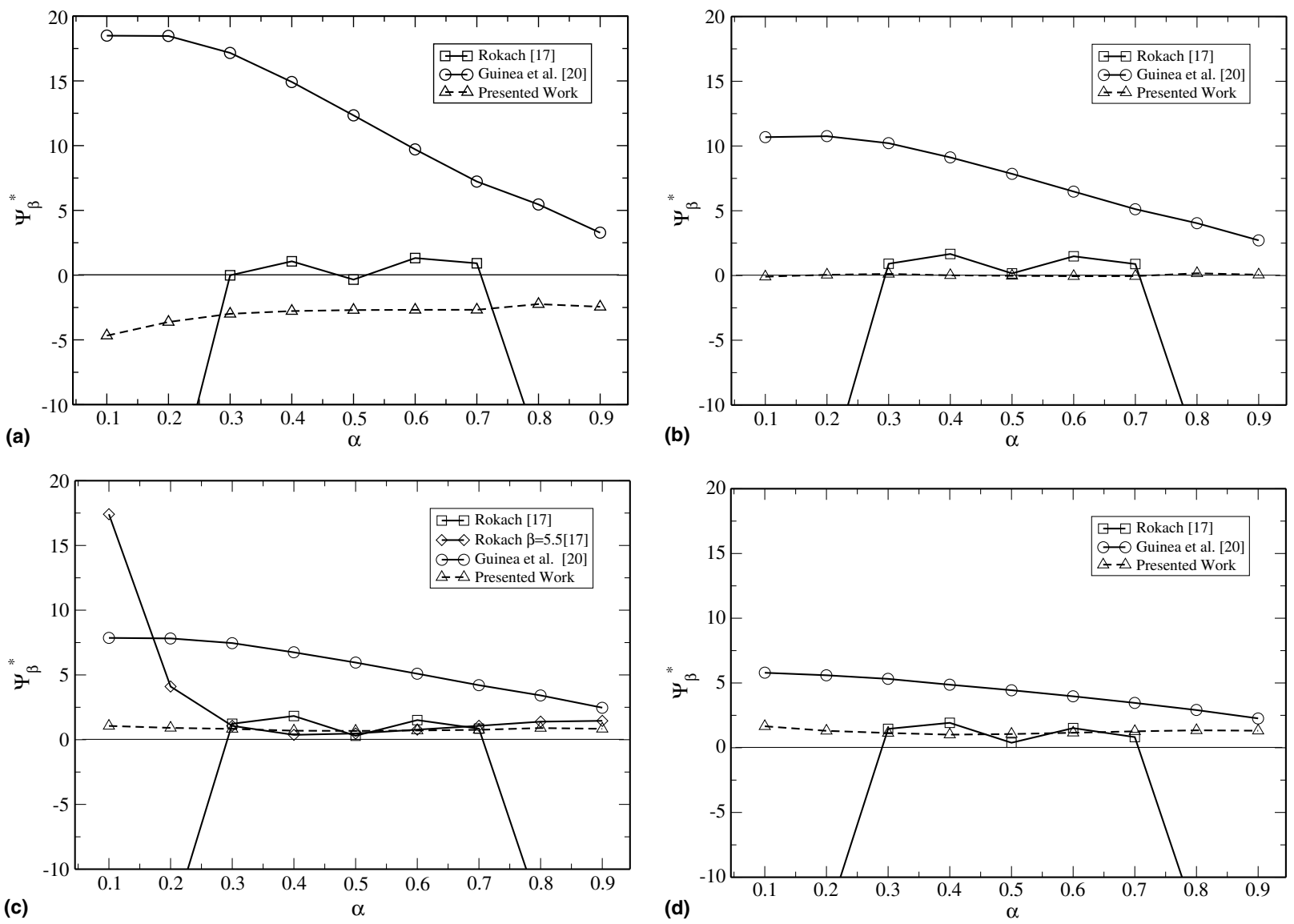

Fig. 5. Deviation of the proposed expression for $\psi_{\beta}^{*}(\alpha)$ relative to various analytical expressions and to FEM calculations. (a) $\beta=2.5$, (b) $\beta=4$, (c) $\beta=5.5$ and (d) $\beta=8$.

- the proposed expression,

- the general expression proposed by Guinea et al. [20] for TPB specimens,

- the general expression proposed by Rokach [17] and

- the specific expression proposed by Rokach for $\beta \quad 5.5$ [17].

Although the problem considered is an OPB test, the results presented by Guinea et al. [20] for TPB specimens are included to quantify the difference when TPB conditions are considered instead of OPB conditions. In this case, the difference decreases as $\alpha$ or $\beta$ increases, ranging between $2 \%$ and $20 \%$.

Rokach [17] noted that the results obtained with his proposed expressions are a very good approximation in a crack length range between $\alpha \quad 0.30$ and $\alpha \quad 0.70$. In the case of $\beta \quad 5.5$, the span-to-width ratio for Charpy specimens, Rokach [17] presented a specific expression with a percentage difference from the results obtained by the FEM as shown in Fig. 5(c).

The expression proposed here is applicable in the range $0.10 \leqslant \alpha \leqslant 0.90$ and was validated for all the values of $\beta$ mentioned above, including $\beta$ 5.5. The difference from the finite element approximation is almost constant (within $2 \%$ ), for every $\alpha$ ranging between 0.10 and 0.90 .

So our general expression to calculate the SIF from the applied load is validated for different values of $\alpha$ and $\beta$, and is a better approximation to the finite element solution than those proposed by other authors, including the particular case of $\beta \quad 5.5$. 


\subsection{SIF from the measure of the CMOD}

Fig. 6 shows, for $\beta \quad 2.5, \beta \quad 4, \beta \quad 5.5$ and $\beta \quad 8$, the percentage difference, $\Lambda_{\beta}^{*}(\alpha)$, between the value of the function $\lambda_{\beta}^{*}(\alpha)$, with which we calculate the SIF from the CMOD in an OPB specimen, obtained by the FEM and:

- the proposed expression and

- the general expression proposed by Guinea et al. [20] for TPB specimens.

The differences from the numerical results are similar for OPB or TPB, below $4 \%$ for $\alpha$ ranging between 0.10 and 0.80 and any $\beta$ considered.

In some practical cases it is difficult to distinguish between TPB and OPB. As shown in Section 5.1, if the SIF of an OPB beam is calculated from the measurement of the applied load, considering TPB conditions, the difference between these results and those obtained considering the real OPB conditions range between $2 \%$ and $20 \%$. However, if the SIF is calculated from the measurement of the CMOD, the maximum difference considering TPB instead of OPB conditions is below 4\%. The reason may be that the influence of the boundary conditions is higher in the load SIF relationship that in the CMOD SIF relationship.
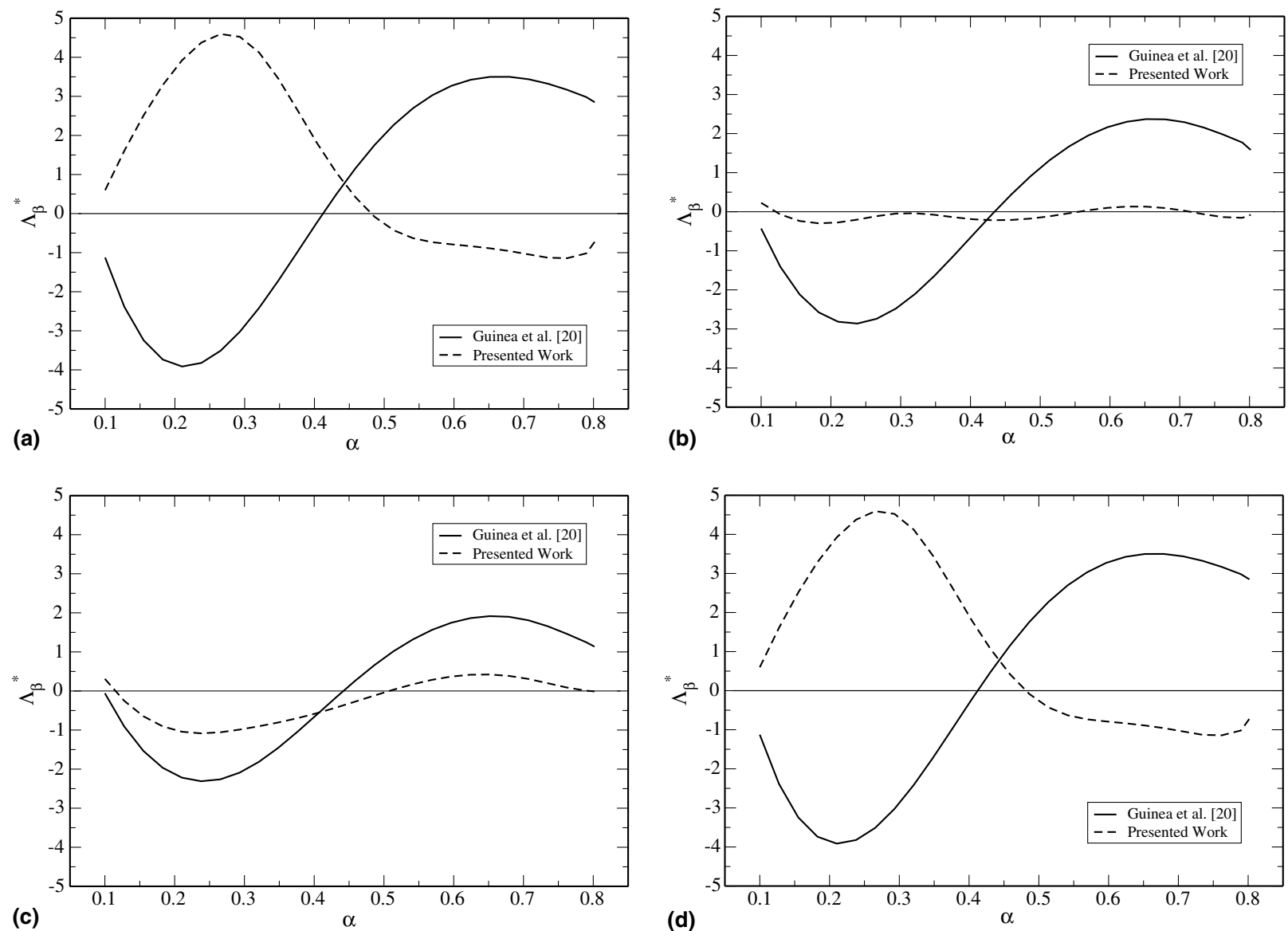

Fig. 6. Deviation of the proposed expression for $\lambda_{\beta}^{*}(\alpha)$ relative to Guinea et al. [20] analytical expression and to FEM calculations. (a) $\beta=2.5$ (b) $\beta=4$ (c) $\beta=5.5$ and (d) $\beta=8$. 
Following the reasoning in the preceding paragraph, for any $\beta$ considered, the SIF of an OPB beam is better calculated from the measurement of the CMOD because the numerical results are similar (below 4\%) for $\alpha$ ranging between 0.10 and 0.80 .

\section{Summary and conclusions}

We present closed-form expressions for the stress intensity factor (SIF) of a general one-point beam-like pre-cracked specimen. From these expressions the SIF can be determined whether from the applied load or from the measurement of the crack mouth opening displacement (CMOD).

The expressions were obtained by decomposition of the general case as the sum of a pure bending, a threepoint bending and a one-point-bending state for a reference span-to-width ratio. The solutions for pure bending and for three-point bending are well established. That of a one-point bend specimen with a span-to-width ratio equal to 4 was obtained in the present work.

The general expressions obtained for the SIF from the applied load were compared to numerical simulations of specimens of different span-to-width ratio and different crack lengths. In all cases, the accuracy was above that developed by other authors [17], fitting the results of $2 \mathrm{D}$ numerical analysis to different values of the crack length and the span-to-width ratio.

In OPB tests, no general formulae for the SIF from the CMOD were encountered. The expressions developed here were compared with numerical simulations of specimens of different span-to-width ratio and different crack lengths. In all cases, the accuracy was higher than the expression for the TPB specimens normally used to analyze dynamic bending tests.

These formulae can be applied to more precise analyses of the dynamic one-point bending tests, without using expressions that are strictly applicable to TPB tests.

\section{References}

[1] Kobayashi T, Yamamoto I, Niimomi M. Evaluation of dynamic fracture toughness parameters by instrumented Charpy impact test. Engng Fracture Mech 1986;24(7):773 82.

[2] Ruiz C, Mines R. The Hopkinson pressure bar: an alternative to the instrumented pendulum for Charpy test. Int J Fracture 1985;29(2):101 9

[3] Yokoyama T. Determination of dynamic fracture initiation toughness using a novel impact bend test procedure. J Press Vessel Technol 1993;115(2):389 97.

[4] Bacon C, Farm J, Lataillade JL. Dynamic fracture toughness determined from load point displacement. Exp Mech 1994;20(1):217 23.

[5] Rubio L, Fernández Sáez J, Navarro C. Determination of dynamic fracture initiation toughness using three point bending tests in a modified Hopkinson pressure bar. Exp Mech 2003;43(4):379 86.

[6] Bohme W, Kalthoff JD. The behaviour of notched bend specimens in impact testing. Int J Fracture 1982;20(4):R139 43.

[7] Crouch BA. Finite element modeling of the three point bend impact test. Comput Struct 1993;48(4):167 73.

[8] Kalthoff JF, Winkler SB, Bohme W, Shockey DA. Mechanical response of cracks to impact loading. In: Brno Czechoslovakia Czechoslovak Academy of Sciences, Institute of Physical Metallurgy, International conference on dynamical properties and fracture dynamics of engineering materials, 1983.

[9] Giovanola JH. Investigation and application of the one point bend impact test. Am Soc Testing Mater, ASTM STP 1986;905:307 28.

[10] Weisbrod G, Rittel D. A method for dynamic fracture toughness determination using short beams. Int J Fracture 2000;104(1):89 103.

[11] Rittel D, Pinneau A, Clisson J, Rota L. On testing of Charpy specimens using the one point bend impact technique. Exp Mech 2002;42(3):247 52.

[12] Giovanola JH, Klopp RW, Kirkpatrick SW. A note on one point bend impact fracture experiments with curve specimens. J Testing Eval 1998;16(1):79 81 .

[13] Bui HD, Maigre H, Rittel D. A new approach to the experimental determination of the dynamic stress intensity factor. Int J Solids Struct 1992;29(23):2881 95.

[14] Beinert J, Kalthoff JF. In: Sih GC, editor. Experimental determination of dynamic stress intensity factors by shadow patterns. Mechanics of fracture, 7. Martinus Nijhoff: La Hague; 1981. p. 281330.

[15] Kishimoto K, Fujino Y, Aoki S, Sakata M. A simple formula for the dynamic stress intensity factor of an impacted freely supported bend specimen. JSME Int J, Series I 1990;33(7):51 6.

[16] Rokach IV. Modal approach for processing one and three point bend test data for DSIF time diagram determination. Part I theory. Fatigue Fracture Engng Mater Struct 1998;21(8):1007 14.

[17] Rokach IV. Modal approach for processing one and three point bend test data for DSIF time diagram determination. Part II calculations and results. Fatigue Fracture Engng Mater Struct 1998;21(8):1015 26. 
[18] Rubio L. Determinación de parámetros de fractura dinámica a alta velocidad de deformación. PhD thesis, Universidad Carlos III de Madrid; 1999.

[19] Loya JA. Efectos tridimensionales en la determinación de la tenacidad de fractura dinámica. PhD thesis, Universidad Carlos III de Madrid; 2004.

[20] Guinea GV, Pastor JY, Planas J, Elices M. Stress intensity factor compliance and CMOD for a general three point bend beam. Int J Fracture 1998;89(3):103 16.

[21] Tada H, Paris P, Irwin G. The stress analysis of cracks handbook. St. Louis, Missouri, USA: Paris Productions Inc; 1985.

[22] ABAQUS. Users Manual Version 6.4. Karlsson and Sorensen Inc, 2003.

[23] Parks DM. The virtual crack extension method for nonlinear material behavior. Comput Meth Appl Mech Engng 1977;12(3):353 64.

[24] Shih CF, Moran B, Nakamura T. Energy release rate along a three dimensional crack front in a thermally stressed body. Int $\mathrm{J}$ Fracture 1986;30(2):79 102. 